Swarthmore College

Works

4-1-1998

\title{
Scaling Studies Of Spheromak Formation And Equilibrium
}

Cameron G. R. Geddes , '97

Thomas W. Kornack , '98

Michael R. Brown

Swarthmore College, doc@swarthmore.edu

Follow this and additional works at: https://works.swarthmore.edu/fac-physics

Part of the Physics Commons

Let us know how access to these works benefits you

\section{Recommended Citation}

Cameron G. R. Geddes, '97; Thomas W. Kornack , '98; and Michael R. Brown. (1998). "Scaling Studies Of Spheromak Formation And Equilibrium". Physics Of Plasmas. Volume 5, Issue 4. 1027-1034. DOI:

10.1063/1.872632

https://works.swarthmore.edu/fac-physics/109

This work is brought to you for free by Swarthmore College Libraries' Works. It has been accepted for inclusion in Physics \& Astronomy Faculty Works by an authorized administrator of Works. For more information, please contact myworks@swarthmore.edu. 


\section{AIP | Physics of \\ Plasmas}

\section{Scaling studies of spheromak formation and equilibrium}

C. G. R. Geddes, T. W. Kornack, and M. R. Brown

Citation: Physics of Plasmas (1994-present) 5, 1027 (1998); doi: 10.1063/1.872632

View online: http://dx.doi.org/10.1063/1.872632

View Table of Contents: http://scitation.aip.org/content/aip/journal/pop/5/4?ver=pdfcov

Published by the AIP Publishing

\section{Articles you may be interested in}

Stable spheromak formation by merging in an oblate flux conserver

Phys. Plasmas 17, 032510 (2010); 10.1063/1.3334324

Dipole trapped spheromak in a prolate flux conserver

Phys. Plasmas 13, 102503 (2006); 10.1063/1.2356690

Sustained spheromak coaxial gun operation in the presence of an $\mathrm{n}=1$ magnetic distortion

Phys. Plasmas 13, 022504 (2006); 10.1063/1.2167911

Plasma diagnostics for the sustained spheromak physics experiment

Rev. Sci. Instrum. 72, 556 (2001); 10.1063/1.1318246

Taylor relaxation and $\lambda$ decay of unbounded, freely expanding spheromaks

Phys. Plasmas 7, 3625 (2000); 10.1063/1.1287137

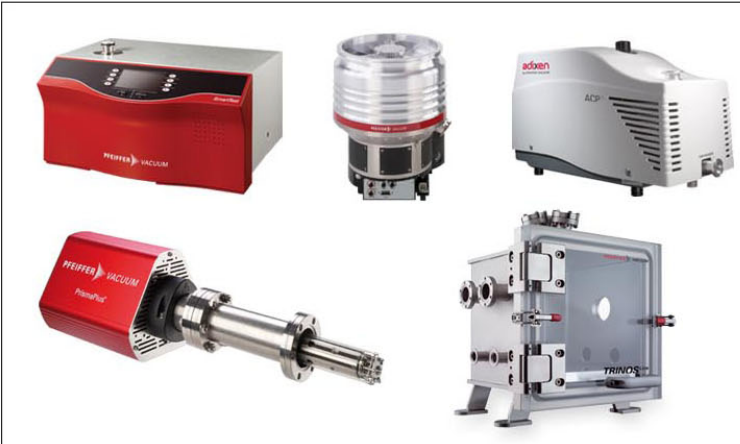

Vacuum Solutions from a Single Source

- Turbopumps

- Backing pumps

- Leak detectors

- Measurement and analysis equipment

- Chambers and components

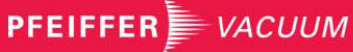




\title{
Scaling studies of spheromak formation and equilibrium
}

\author{
C. G. R. Geddes, T. W. Kornack, and M. R. Brown ${ }^{\text {a) }}$ \\ Department of Physics and Astronomy, Swarthmore College, Swarthmore, Pennsylvania 19081-1397
}

(Received 2 October 1997; accepted 23 December 1997)

\begin{abstract}
Formation and equilibrium studies have been performed on the Swarthmore Spheromak Experiment (SSX). Spheromaks are formed with a magnetized coaxial plasma gun and equilibrium is established in both small $\left(d_{\text {small }}=0.16 \mathrm{~m}\right)$ and large $\left(d_{\text {large }}=3 d_{\text {small }}=0.50 \mathrm{~m}\right)$ copper flux conservers. Using magnetic probe arrays it has been verified that spheromak formation is governed solely by gun physics (in particular the ratio of gun current to flux, $\mu_{0} I_{\text {gun }} / \Phi_{\text {gun }}$ ) and is independent of the flux conserver dimensions. It has also been verified that equilibrium is well described by the force free condition $\nabla \times \mathbf{B}=\lambda \mathbf{B}(\lambda=$ constant), particularly early in decay. Departures from the force-free state are due to current profile effects described by a quadratic function $\lambda=\lambda(\psi)$. Force-free SSX spheromaks will be merged to study magnetic reconnection in simple magnetofluid structures. (C) 1998 American Institute of Physics. [S1070-664X(98)00204-3]
\end{abstract}

\section{INTRODUCTION}

A spheromak is a toroid of plasma with toroidal and poloidal magnetic fields of comparable strength generated by currents flowing in the plasma, and with no material linking the center of the torus (Figure 1). The unique properties of spheromaks have recently fueled interest in their use for studies of magnetic reconnection ${ }^{1-5}$ and magnetic confinement fusion. ${ }^{6,7}$ There has been a recent renaissance in spheromak research beginning with the assertion by Fowler and Hooper ${ }^{8-10}$ that spheromaks generated by the Los Alamos Compact Torus Experiment (CTX) group ${ }^{6}$ may have had good core confinement during decay. Fowler's argument is that most of the Ohmic power from a magnetized plasma gun went to the cool, resistive edge plasma and furthermore that magnetic decay is regulated by flux at the edge. These two points conspire to make a poor global confinement time $\tau_{E}$ dominated by edge physics. The highest performance CTX spheromaks were gun-produced and formed in closefitting $0.56 \mathrm{~m}$ diameter copper flux conservers. Typical best parameters were $T_{e}=400 \mathrm{eV}, n_{e}=5 \times 10^{20} \mathrm{~m}^{-3}$ and $B_{\text {wall }}$ $=3 T^{11,12}$ and produced significant $\mathrm{x}$-rays from runaway electrons. ${ }^{13}$ Hooper suggested that the $\mathrm{x}$-rays were evidence of closed flux surfaces in the core and that poor global confinement was due to open flux at the edge.

The goal of the Swarthmore Spheromak Experiment (SSX) is to study the basic physics of the spheromak and to use stable spheromaks as force-free reservoirs of magnetic flux for merging and reconnection experiments (see Figure 2 ). We have performed experiments at SSX in both small $\left(d_{\text {small }}=0.16 \mathrm{~m}\right)$ and large $\left(d_{\text {large }}=0.50 \mathrm{~m}\right)$ flux conservers using coaxial plasma guns in a high vacuum, low impurity environment. The planned Sustained Spheromak Physics Experiment (SSPX) at Lawrence Livermore National Laboratory will study the assertions of Fowler and Hooper further in a sustained, steady state discharge. The SSPX spheromak will be formed in a $1.0 \mathrm{~m}$ diameter copper flux conserver

${ }^{a)}$ Electronic mail: mbrown3@swarthmore.edu using coaxial magnetized plasma guns in a high vacuum, low impurity environment. It is our hope that scaling studies on SSX can be applied to SSPX design and operation.

This paper describes spheromak formation and equilibrium experiments on SSX. In section II A a simple theory of spheromak formation is presented, in section II B spheromak equilibrium theory and numerical modelling results are presented. Section III describes the SSX spheromak experiment. In section III A formation results from both flux conservers are presented and in section III B equilibrium results from both flux conservers are presented. Section IV is a conclusion and overview of the results. Details of probe calibration and design are presented in an appendix.

\section{THEORY}

\section{A. Formation}

Spheromaks are formed in SSX by a magnetized coaxial plasma gun. ${ }^{14,15}$ Magnetic flux (called the "stuffing flux") is deposited in the inner electrode of the gun using an external coil. High purity hydrogen is puffed into the annular gap between the inner and outer electrode. A high voltage (up to $10 \mathrm{kV}$ ) is applied which ionizes the gas and creates a radial current sheet. The discharge current (over $100 \mathrm{kA}$ ) generates toroidal flux and the axial $J \times B$ force ejects plasma out of the gun. If the $J \times B$ force exceeds the magnetic tension of the stuffing flux then a free spheromak is formed. The process is analogous to the blowing of a soap bubble. The soap film tension is analogous to the stuffing flux tension, while the pressure of one's breath in forming the soap bubble is akin to the magnetic pressure of the gun current.

Spheromak formation by magnetized coaxial plasma gun has been discussed both theoretically and experimentally. ${ }^{14-17}$ The fundamental idea in all this work is that a threshold value of $\lambda_{\text {th }}=\mu_{0} I_{\text {gun }} / \Phi_{\text {gun }}$ must be exceeded in order that a spheromak is formed. The dimensions of $\lambda_{t h}$ are an inverse length so one expects the threshold parameter to be some constant of order unity divided by the scale of the system. Sophisticated theories ${ }^{18}$ predict that for a 


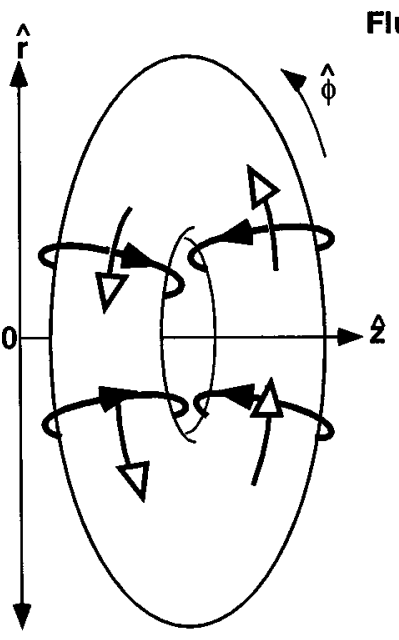

(a)
Flux Conserver

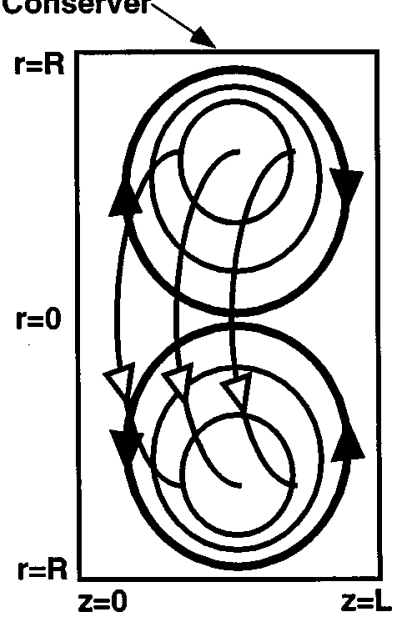

(b)

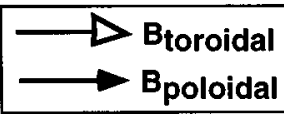

Bpoloidal
FIG. 1. Two views of a spheromak with the magnetic fields and coordinate axes indicated. The cross section at right is taken in the poloidal $(r-z$ plane). The flux conserver is shown in the cross section view only.

cylindrically symmetric gun $\lambda_{t h}=3.83 / r_{\text {gun }}$ where 3.83 is the first zero of the Bessel function $J_{1}$. Note that $\lambda_{t h}$ depends only on gun geometry in this model. For SSX, 3.83/ $r_{\text {gun }}$ $=46 \mathrm{~m}^{-1}$ where $r_{\text {gun }}=0.083 \mathrm{~m}$.

A simple formation theory can be constructed by assuming a thin radial current sheet that is free to move axially and a purely radial stuffing flux (see Figure 3). Force balance on the current sheet requires that the magnetic tension of the stuffing flux equals the net $J \times B$ force. Since the gun current produces an azimuthal field $B_{\theta}=\mu_{0} I_{g u n} / 2 \pi r$ we can write the magnetic pressure on the back of the sheet as:

$$
P_{B}=\frac{B_{\theta}^{2}}{2 \mu_{0}}=\frac{\mu_{0} I_{\text {gun }}^{2}}{8 \pi^{2} r^{2}} .
$$

If we integrate this pressure over the annular face of the current sheet we find for the net $J \times B$ force:

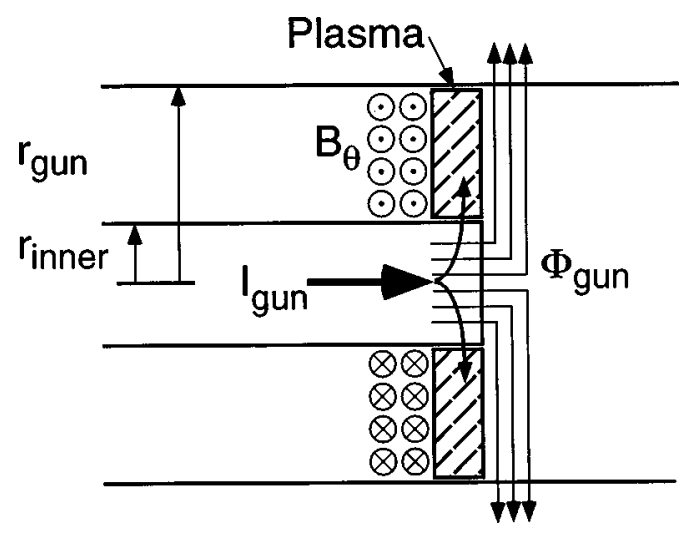

FIG. 3. Spheromak formation geometry.

$$
F=\frac{\mu_{0} I_{\text {gun }}^{2}}{4 \pi} \ln \left(r_{\text {gun }} / r_{\text {inner }}\right) .
$$

Now if the stuffing flux is distended an amount $\delta z$ by the magnetic pressure $P_{B}$, then the work done by this force equals the increase in magnetic energy: $F \delta z=\Delta W_{\text {mag }}$ $=\left(B_{\text {stuff }}^{2} / 2 \mu_{0}\right)\left(\pi r_{\text {inner }}^{2}\right) \delta z$. Noting that $\Phi_{\text {gun }}=B_{\text {stuff }} \pi r_{\text {inner }}^{2}$ and solving for $\lambda$ we find:

$$
\lambda_{\text {th }}=\frac{\mu_{0} I_{\text {gun }}}{\Phi_{\text {gun }}}=\frac{1}{r_{\text {inner }}} \sqrt{\frac{2}{\ln \left(r_{\text {gun }} / r_{\text {inner }}\right)}} .
$$

Interestingly, this expression also yields $\lambda_{t h}=46 \mathrm{~m}^{-1}$ for our parameters $\left(r_{\text {gun }}=0.083 \mathrm{~m}\right.$ and $\left.r_{\text {inner }}=0.031 \mathrm{~m}\right)$.

\section{B. Equilibrium}

Immediately following formation, the spheromak relaxes to a minimum energy state subject to the constraints of constant magnetic helicity and zero magnetic flux $(\Psi=0)$ at the conducting wall. ${ }^{19-21}$ The steady state spheromak equilibrium is characterized by:

$$
\nabla P=\mathbf{J} \times \mathbf{B} .
$$

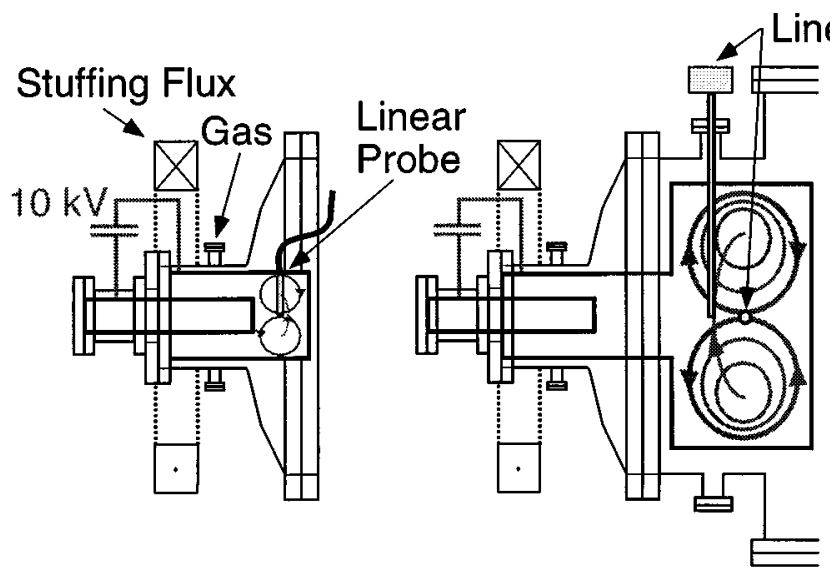

(a)

(b)

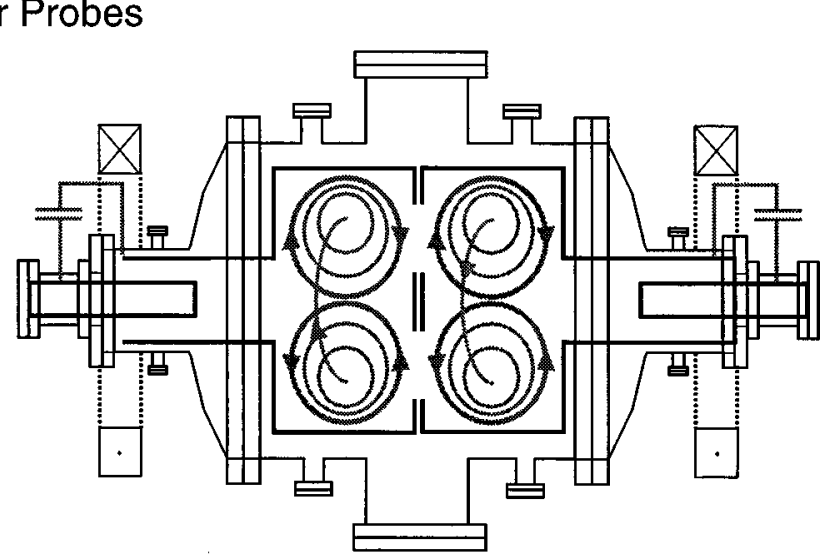

(c)

FIG. 2. A schematic of the SSX gun showing (a) small and (b) large flux conservers and the magnetic probes for formation and equilibrium measurements. (c) shows both guns with two large flux conservers to allow reconnection studies. 
This is simply an expression of static pressure balance between gradients in kinetic pressure and magnetic forces (the general form of the Grad Shafranov equation). Spheromaks are typically characterized by low $\beta$ (the ratio of plasma pressure to magnetic pressure), so the simplest equilibrium model is then given by letting $\nabla P=0$ in (2) above. In this case, the equilibrium equation reduces to a simple form:

$$
\nabla \times \mathbf{B}=\lambda \mathbf{B},
$$

where $\lambda$ is an inverse length and is in general a function of the poloidal flux $\Psi$.

Constant $\lambda$ corresponds to the minimum energy, or force free, state for the spheromak, and this simple model is often an applicable one. ${ }^{19,20}$ The constant- $\lambda$ force free equation can be solved directly with the boundary conditions of a closed perfectly conducting right cylinder, giving an analytical solution: ${ }^{18}$

$$
\begin{aligned}
& B_{r}=B_{0} \frac{k_{z}}{k_{r}} J_{1}\left(k_{r} r\right) \cos \left(k_{z} z\right), \\
& B_{t}=B_{0} \frac{\lambda}{k_{r}} J_{1}\left(k_{r} r\right) \sin \left(k_{z} z\right), \\
& B_{z}=B_{0} J_{0}\left(k_{r} r\right) \sin \left(k_{z} z\right), \\
& \Psi=B_{0} \frac{r}{k_{r}} J_{1}\left(k_{r} r\right) \sin \left(k_{z} z\right),
\end{aligned}
$$

where $B_{0}$ is an arbitrary constant, and with:

$$
k_{z}=\frac{\pi}{L}, \quad k_{r}=\frac{3.8317}{R}, \lambda=\sqrt{k_{r}^{2}+k_{z}^{2}},
$$

where $R$ is the radius and $L$ the length of the conserver. For the SSX case, $\lambda_{S F C}=55 \mathrm{~m}^{-1}$ for the small flux conserver and $\lambda_{L F C}=18 \mathrm{~m}^{-1}$ for the large flux conserver. Since $\mu_{0} \mathbf{J}=\nabla \times \mathbf{B}$, then by (3):

$$
\mathbf{J}=\frac{\lambda}{\mu_{0}} \mathbf{B}
$$

so that $\mathbf{J}$ is proportional and parallel to $\mathbf{B}$. Constant $\lambda$ indicates a flat current profile, since $J / B$ is constant, and hence $I_{z}=\int \mathbf{J} \cdot \mathbf{d A}$ is proportional to $\Psi=\int \mathbf{B} \cdot \mathbf{d A}$. Also characteristic of this solution is that the magnetic axis is located at $r$ $=0.63 R$, where $R$ is the radius of the flux conserver.

Variable $\lambda$ states can be used to model spheromaks with non uniform current distributions, which depart from the force-free state. When considering variable $\lambda$ states, it is convenient to express $\lambda$ as a power series in $\Psi$, and usually only the first order term is relevant. This yields: ${ }^{22-24}$

$$
\lambda=\bar{\lambda}\left(1+\alpha\left(2 \frac{\Psi}{\Psi_{\max }}-1\right)\right),
$$

where $\alpha$ governs the dependence on $\Psi$, and where $\bar{\lambda}$ is the average value of $\lambda$ over the plasma. If $\alpha$ is positive, then the current profile is peaked which is typical of spheromaks in decay, since the edges are cooler and more resistive than the core. Negative $\alpha$ corresponds to hollow current profiles, typical of spheromaks still being driven by the gun, while zero $\alpha$ is the fully relaxed force free state corresponding to constant $\lambda$. A similar convention can be used when second order in $\Psi$ is desired, yielding:

$$
\lambda=\bar{\lambda}\left(1+\alpha\left(2 \frac{\Psi}{\Psi_{\max }}-1\right)+\gamma\left(2\left(\frac{\Psi}{\Psi_{\max }}\right)^{2}-1\right)\right) .
$$

The process can of course proceed to arbitrary order in $\Psi$ as needed. Higher order in $\Psi$ allows description of more sharply peaked current distributions. We have found that a quadratic form of $\lambda(\Psi)$ is sufficient to fit our experimental data.

When it is neccessary to consider the effects of plasma pressure on the equilibrium (generally when $\beta>10 \%$ ), a more general description is needed. The Grad Shafranov equation can be written in cylindrical coordinates:

$$
\nabla \cdot\left(\frac{1}{r^{2}} \nabla \Psi\right)+4 \pi^{2} \mu_{0} P^{\prime}+\frac{\mu_{0}^{2}}{r^{2}} I_{z} I_{z}^{\prime}=0,
$$

where $P$ and $I_{z}$ are functions of $\Psi$ and primes indicate derivatives with respect to $\Psi$. Because it involves three independent quantities, $\Psi, P(\Psi)$, and $I_{z}(\Psi)$, the Grad Shafranov equation does not uniquely determine the equilibrium. In order to use the equation, two of the functions (usually $P(\Psi)$ and $I_{z}(\Psi)$ ) are specified and the remaining one can be solved for. We typically use forms for $I_{z}$ and $I_{z}^{\prime}$ which result from Eq. (5) and the expressions for $\lambda$ [Eqs. (6) and (7)].

A range of solutions have been calculated for both flux conserver geometries and with both linear and quadratic current profiles in $\Psi$. We determine the models which best fit our experimental data by trial and error. Examination of a few solutions demonstrates the effects of various current profiles and flux conserver shapes on the equilibrium.

First, we consider the effects of device geometry, using the force-free solution as an illustration. Figure 4 shows three solutions generated in the two SSX flux conservers (illustrated in Figure 2) and in a "perfect" closed cylinder geometry. The closed cylinder and large flux conserver solutions are negligibly close to one another. Agreement between the analytical and simulated solutions is found in these conservers. $\lambda_{0}=18.4 \mathrm{~m}^{-1}$ for the large flux conserver, in agreement with the analytic solution's formula for $\lambda$ [see Eq. (4)]. This confirms that the solver is working properly. In contrast, the small flux conserver is not a good approximation of a closed cylinder geometrically. Most importantly, the spheromak does not center in the small conserver, and flux protrudes back into the gun. The magnetic axis sits at $z$ $=0.42 L$, rather than at $z=0.5 \mathrm{~L}$ as it does in both the perfect and large flux conserver geometries. This means that a probe placed at $z=0.5 \mathrm{~L}$ will see a small nonzero $B_{r}$ in the small flux conserver, but zero $B_{r}$ in the others. In addition, though a full stability analysis was not performed, we note that the small flux conserver spheromak may have a tendency to tilt back into the gun since $\lambda_{S F C}>\lambda_{t h}$.

Next, we consider the effects of various current and pressure profiles. We expect the force-free (constant $\lambda$ ) state to appear following the spheromak's initial relaxation, since the relaxation process tends towards minimum energy. This solution is characterized by a magnetic axis at $0.63 R$. Vari- 


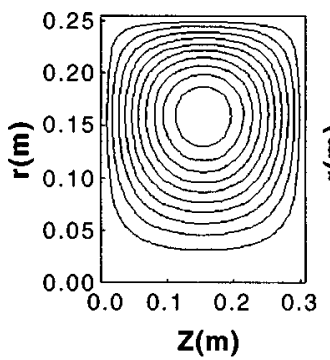

(a)

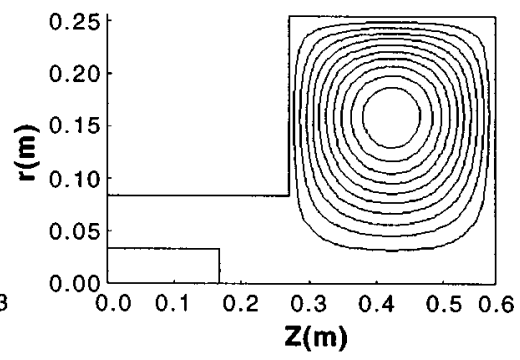

(b)

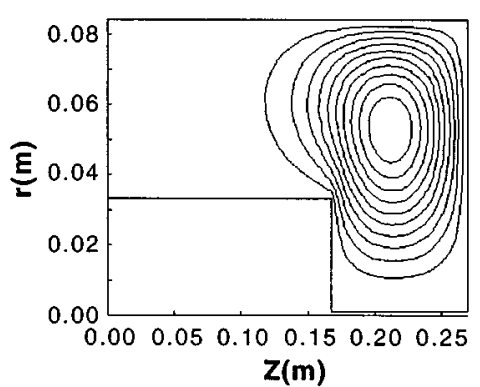

(c)
FIG. 4. Effects of geometry on the solution. Solutions for the poloidal flux (a) in a perfect can, and (b) in the SSX large conserver are similar, while a solution (c) in the small conserver is distorted, protruding more into the gun region.

able $\lambda$ states with positive $\alpha$, corresponding to peaked current distributions, can account for spheromaks in decay. Some trial and error is needed to determine the resonant value of $\bar{\lambda}$ in these cases, since it is not exactly equal to the constant $\lambda$ value. When first order positive $\alpha$ states are computed, one can observe the shifting of the magnetic axis out from $0.63 R$ (the constant $\lambda$ value) to $.67 R$ at $\alpha=0.98$. Second order solutions push the magnetic axis out still farther. Plots of several representative solutions are shown in Figure 5. We do not sustain the spheromak, so that detachment from the gun occurs early and the plasma settles rapidly into a constant $\lambda$ state after formation rather than a negative $\alpha$ state which might correspond to current continuing to be driven on the outer flux surfaces. Figures 5a and c (constant $\lambda$ and quadratic $\lambda$ ) represent the best equilibrium fits to data presented in section III B.

We have generated non-uniform pressure profiles but found that we were unable to fit them to our data. High $\beta$ states moved the magnetic axis out radially as expected but degraded other aspects of the fit. Since the derivative of flux is determined by both pressure and current, adding pressure increases $\Psi_{\max }$ for a given $I_{z}$ distribution. Since $B_{t}$ depends only on $I_{z}$ while $B_{p}$ depends on $\Psi$, this means that while force free solutions have roughly equal toroidal and poloidal field magnitudes, finite pressure solutions have relatively more poloidal field. Pressure effects can be seen when $\beta$ exceeds about $10 \%$. Below this point, they are not distinguishable.

\section{EXPERIMENTAL RESULTS}

Measurements of spheromak formation and equilibrium have been performed at the Swarthmore Spheromak Experiment (SSX). Identical magnetized, tungsten coated plasma

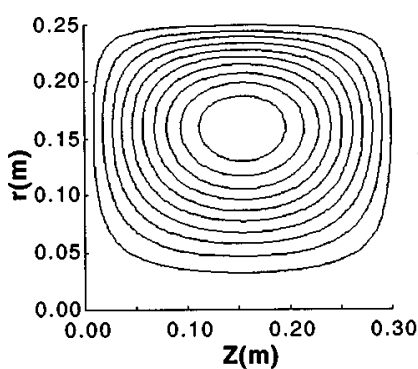

(a)

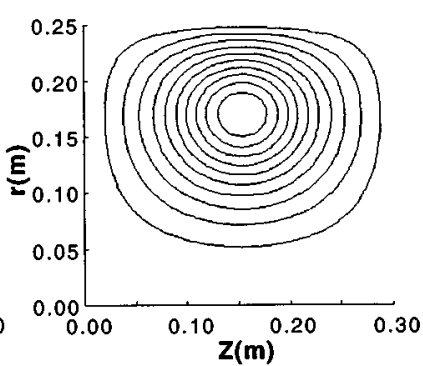

(b)

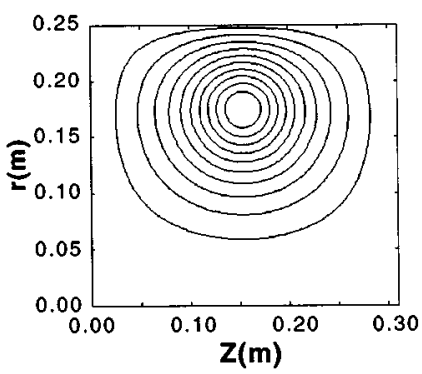

(c)

FIG. 5. Zero pressure solutions for the poloidal flux with various $\lambda$ profiles. (a) Constant $\lambda$ appropriate for early in decay, (b) linear $\lambda(\Psi)$ and (c) quadratic $\lambda(\Psi)$ with $\bar{\lambda}=29 \mathrm{~m}^{-1}, \alpha \approx 0.22$, and $\gamma \approx 0.75$ appropriate for late in decay.

guns $\left(r_{\text {gun }}=0.083 \mathrm{~m}\right.$ and $\left.r_{\text {inner }}=0.031 \mathrm{~m}\right)$ can be used to independently form spheromaks in either small $r_{\text {small }}=r_{\text {gun }}=0.083 \mathrm{~m}$ or large $r_{\text {large }}=3 r_{\text {small }}=0.25 \mathrm{~m}$ copper flux conservers (see Figure 2). In addition, guns can be fired simultaneously into separate flux conservers for reconnection experiments. For the experiments discussed here, $L_{\text {small }}=0.102 \mathrm{~m}$ and $L_{\text {large }}=0.305 \mathrm{~m}$ so that $L / r$ is close to 1.22 in both cases. These dimensions satisfy the requirement for stability against the tilt mode: $L / r<1.67^{25,26}$ though the small conserver may still be unstable for other reasons. The gun dimensions are identical in both cases to facilitate scaling studies and comparisons.

The guns are powered by identical $10 \mathrm{kV}, 25 \mathrm{~kJ}$ capacitive power supplies $(5 \mathrm{kV}, 6 \mathrm{~kJ}$ typical). A separate system provides up to $4 \mathrm{mWb}$ of stuffing flux. Typical SSX spheromak densities are $n_{e} \leqslant 10^{15} \mathrm{~cm}^{-3}$ in the small flux conserver and $n_{e} \leqslant 10^{14} \mathrm{~cm}^{-3}$ in the large flux conserver (from Alfvén speed and particle inventory estimates). Triple Langmuir probe measurements in the large flux conserver confirm that $n_{e}=5 \times 10^{13} \mathrm{~cm}^{-3}$ and show $T_{e} \cong 20 \mathrm{eV}$ so that $\beta \leqslant 0.1$.

The magnetic probes used for equilibrium measurements consist of two linear arrays mounted in the SSX large flux conserver or one in the small conserver as shown in Figure 2. Each array in the large conserver consists of 11 sets of three orthogonal coils in a stainless steel housing. In the small flux conserver, a smaller probe with 5 coilsets is used. The stainless steel casings are thin enough so that the flux diffusion time is short compared to relevant measurements (less than $0.1 \mu \mathrm{s})$ and does not affect the probes. Each coilset measures three axes simultaneously, so that vector $\mathbf{B}$ is measured at 11 radial locations as well as at two locations along the length of the machine and around it toroidally, for a total of 22 simultaneous measurements. Radial sensitivity is empha- 
(a)
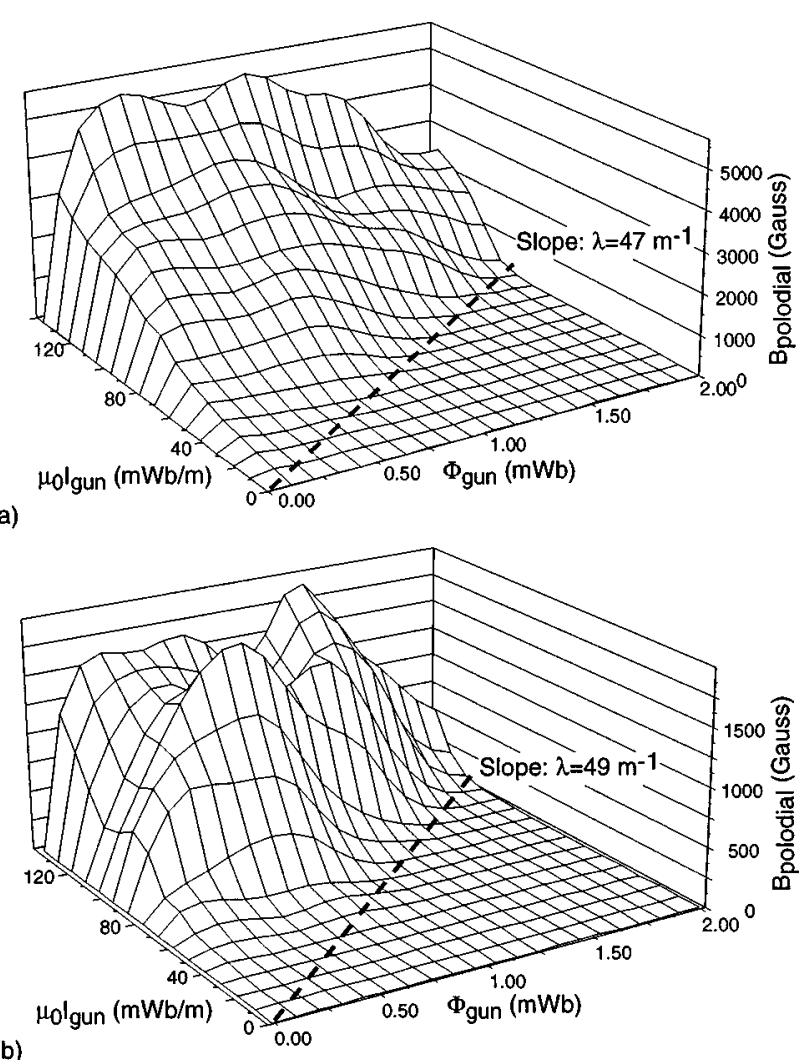

FIG. 6. Formation threshold data. (a) Small flux conserver, (b) Large flux conserver with $\lambda_{t h} \approx 48 \mathrm{~m}^{-1}$ in both cases.

sized since it is most crucial to determining the shape of the equilibrium, while some $z$ resolution is often helpful in order to allow us to distinguish equilibria which differ from one another mostly away from the symmetry axis. Details of probe calibration and design are given in the appendix.

\section{A. Formation}

Scans of $B_{z}$ magnetic data were taken at the edge of both flux conservers in order to determine the formation threshold and optimum operating parameters. Data were taken from $\Phi_{\text {gun }}=0$ to $2.0 \mathrm{mWb}$ at $0.25 \mathrm{mWb}$ intervals and from $I_{\text {gun }}=0$ to $100 \mathrm{kA}$ at $10 \mathrm{kA}$ intervals (a $9 \times 11$ matrix). Averages of several shots were taken each operating point. In Figure 6 we present the results of the scans.

Note first that the peak magnetic fields in the small flux conserver $(5 \mathrm{kG})$ are about a factor of 3 higher than in the large flux conserver $(1.5 \mathrm{kG})$ for the same gun parameters. If energy is conserved, we expect $B^{2}$ to scale like $r^{3}$ or $\left(B_{\text {small }} / B_{\text {large }}\right)^{2}=\left(r_{\text {large }} / r_{\text {small }}\right)^{3}$. However, this is an overestimate since we also expect the relaxation process to be less efficient in the large flux conserver by another factor of $r_{\text {large }} / r_{\text {small }}{ }^{14,27}$ so we expect $B$ to scale roughly like $\mathrm{r}$. For $\mathrm{SSX}, \quad B_{\text {small }} / B_{\text {large }}=3.3$ and $r_{\text {large }} / r_{\text {small }}=3.0$ verifying this scaling.

Next, we note that there is a striking threshold in both flux conservers at $\lambda_{t h}=\mu_{0} I_{\text {gun }} / \Phi_{\text {gun }} \cong 48 \mathrm{~m}^{-1}$ close to the value of $\lambda_{t h}=46 \mathrm{~m}^{-1}$ predicted in section II A. If $\Phi_{\text {gun }}$ is too high and $I_{\text {gun }}$ too low then no spheromak is formed and

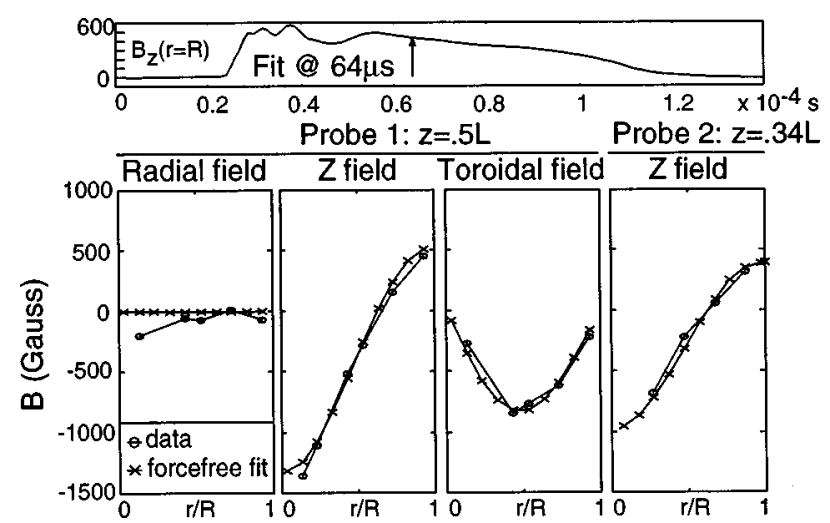

FIG. 7. Force free equilibrium data in the large flux conserver. Early in decay (64 $\mu \mathrm{s}$ ) with flux function as depicted in Figure 5(a).

no magnetic signal is recorded. This threshold does not scale with the dimensions of the flux conserver attached to the gun and depends only on gun dimensions.

A few other features are worth noting. We find that as $\Phi_{\text {gun }} \rightarrow 0$ the spheromak fields vanish (even for large $I_{\text {gun }}$ ). We took an extra set of data at $\Phi_{\text {gun }}=0.1 \mathrm{mWb}$ to verify this observation. This is understandable since as $\Phi_{\text {gun }} \rightarrow 0$ the injected helicity vanishes and a finite helicity object like a spheromak cannot be formed. ${ }^{28}$ In the large flux conserver we find that the spheromak fields vanish at small but finite $I_{\text {gun }}$ and $\Phi_{\text {gun }}$ (even with $\lambda>\lambda_{t h}$ ). This is a reproducible result for which we have no explanation.

\section{B. Equilibrium}

Most equilibrium data have been collected in the large flux conserver, and this is where the most interesting and relevant equilibria are observed. This conserver is also identical to that which will be used for reconnection experiments, so the results apply directly to characterizing the reconnection flux reservoir. A typical shot, which displays the main characteristics observed, is described in detail, followed by a discussion of trends in the data.

A representative shot is shown in Figures 7 and 8. This spheromak was fired in the large flux conserver with $5 \mathrm{kV}$ on the gun bank, $1.5 \mathrm{mWb}$ of stuffing flux and about $100 \mathrm{kA}$ of peak gun current, a setting which was observed to produce
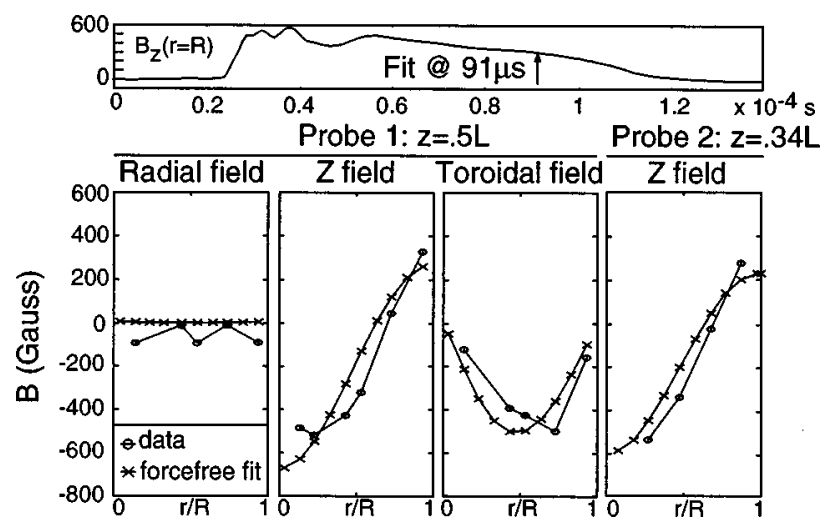

FIG. 8. Quadratic $\lambda$ profile data in the large flux conserver. Late in decay (91 $\mu \mathrm{s})$ with flux function as depicted in Figure 5(c). 
long lived, stable spheromaks. This shot displays the two characteristic phases: constant $\lambda$ force-free state in early decay, and a quadratic $\lambda$ state late in decay.

After a turbulent relaxation phase, the spheromak settles into a state which is a very good fit to the force free constant $\lambda$ state calculated above. Our numerical fit to the experimental data verifies the analytical prediction of $\lambda \approx 18 \mathrm{~m}^{-1}$ [Eq. (4)]. $\lambda$ varies only by about $10 \%$ across the machine. Pressure is also small relative to magnetic field $(\beta \leqslant 10 \%)$. This phase lasts from approximately 52 to $67 \mu \mathrm{s}$ and is shown in Figure 7. It occurs significantly after the peak fields, which is to be expected since the turbulent relaxation phase should dissipate some of the magnetic energy. In this phase, the poloidal field integrates out very close to zero, indicating that flux contours are closed and the spheromak is fully formed and detached from the gun. Toroidal and poloidal field are very close in magnitude, indicating that the relaxation of toroidal into poloidal flux has occurred and that the state is very close to zero pressure. Further, as time passes, the field profiles change only slowly and continuously, indicating that quasi-static equilibrium is established. Also in this phase, radial field decreases to about $10 \%$ of $B_{z}$ with the occasional exception of the $r=0$ reading. This indicates that the spheromak is approximately centered in the conserver. Small deviations from zero radial field are likely due to integration errors. There are no oscillations in the magnetic fields, indicating that the spheromak is force-free and stable. The spheromak now begins slow decay.

As time progresses, the magnetic axis moves outward in radius from $0.63 R$ to $0.71 R$, as illustrated in Figure 8. The peak in toroidal field also moves outward. Two factors may contribute to this effect. If pressure effects are becoming significant, this could move the magnetic axis outward and produce the observed effect. This may be reasonable since the plasma is being resistively heated through its lifetime, potentially increasing pressure effects at the same time magnetic fields are becoming weaker. Alternatively, we may be observing a peaked current state with nonconstant $\lambda$. If increased resistance in the cool edges of the plasma causes current to fall off faster there, which is likely, then we will end up with a peaked current profile state as described above. If so, it must be a second order state, since no first order force free solution has a magnetic axis at such large $r$. The observed profile may also be a combination of these effects. Since the toroidal and poloidal field magnitudes remain comparable, however, it is likely that pressure effects are small. A full fit to models verifies that it is not possible that pressure effects can cause the magnetic axis movement. The numerical solution also yields $P^{\prime} \approx 5 \times 10^{5} \mathrm{~Pa} /$ Weber, corresponding to a $\beta \leqslant 10 \%$. This is likely to be too small to have a significant effect on the equilibrium. A numerical fit shows that a quadratic lambda profile (peaked current distribution) model is the best fit to the data, with $\bar{\lambda}=29 \mathrm{~m}^{-1}, \alpha \approx 0.22$, and $\gamma \approx 0.75$, confirming the expectation of quadratic $\lambda$ and low pressure. This equilibrium was presented in Figure 5. Since we cannot satisfactorily fit finite pressure states to our data we conclude that our $\beta$ must be less than $10 \%$, and this is confirmed by triple probe data.
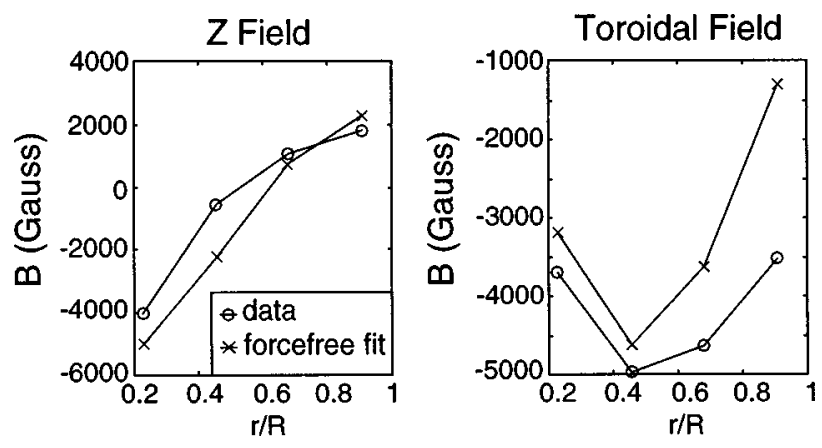

FIG. 9. Small flux conserver equilibrium data.

Magnetic field profiles were measured in the small flux conserver in order to verify scaling and flux conserver shape effects. We found that although the fit was close to the minimum energy state for part of the discharge (Figure 9), the magnetic axis was at approximately $r=0.5 R$ and a stable equilibrium was never reached. The spheromak in the small flux conserver lived only about 20-40 $\mu$ s (about the predicted $L / R$ time), and never settled into a state which could be matched by a reasonable pressure or current distribution. We also found that the radial field was larger than expected, even including expected distortion due to the (relatively) larger opening into the gun. There are several possible explanations for the poor performance of spheromaks in the small flux conserver. First, our simulations show that substantial flux protrudes back into the gun entrance (see Figure 4c). Geometrical perturbations from the gun opening could severely degrade the equilibrium. Second, we could be observing a tilt instability as the the spheromak dynamically falls back into the gun. Third, since the lifetime is short, there is still significant current flowing in the gun. Perturbations from gun current could therefore be affecting equilibrium. Finally, static fringe fields from the stuffing flux could also affect equilibrium in the small flux conserver.

\section{CONCLUSION}

We have used magnetic probe arrays to characterize formation and equilibrium of spheromaks of two different sizes at SSX. Our main conclusions are (1) we have verified that the spheromak formation threshold $\lambda_{t h}=\mu_{0} I_{\text {gun }} / \Phi_{\text {gun }}$ is independent of the dimensions of the flux conserver attached to the gun. (2) The peak magnetic field in the spheromak appears to scale like the inverse of the flux conserver radius for the same gun parameters. (3) Equilibria following formation (at least in the large flux conserver) are well characterized by a constant $\lambda$ and a uniform current profile. (4) Late in decay, departures from constant $\lambda$ are well characterized by a $\lambda$ profile quadratic in $\Psi$. (5) We see no evidence of finite pressure effects.

These results can be compared to those of other researchers who have studied spheromak equilibrium. Kitson and Browning ${ }^{23}$ and $\mathrm{Knox}^{22}$ have both seen evidence of variable $\lambda$ states in decaying spheromaks, but found that only first order variable $\lambda$ states were distinguishable. In contrast, 
we find strong evidence for quadratic profiles in some cases. Wysocki ${ }^{29}$ and $\mathrm{Hart}^{30}$ have seen evidence for significant pressure effects, which we do not observe.

\section{APPENDIX A: PROBE CALIBRATION}

Substantial attention was given to ensuring that the magnetic probes used in these experiments were as accurate as possible. In this appendix, we describe the methods used to avoid cross coupling of signals, to obtain accurate integration of fast magnetic signals, and evaluate probe perturbation on the plasma.

Irregularities in winding and flexibility of the coil form, especially at the very small coil sizes required to minimize plasma disruption, result in cross-coupling between axes on the order of $10 \%$. This can cause significant problems when the signal on one axis is very much larger than that on another, since the coupled signal from the stronger axis will completely overwhelm the desired signal on the weaker one. This is a problem for instance at the edge of the flux conserver or at $z=L / 2$, where $B_{r} \approx 0$, but $B_{z}$ and $B_{t}$ are large. A technique has been devised which allows recovery of signal without cross coupling to within $<1 \%$. After assembly but before insertion, a Helmholtz coil is used to apply a known field along each axis to the probe, and the response of each sensor coil in signal per unit $\dot{B}$ on each axis is determined, giving for each coilset a matrix such that:

$$
K \dot{B}=S,
$$

where $\dot{B}$ is the time derivative of the magnetic field vector, $S$ is the signal vector, and $K$ is a matrix such that $K_{i j}$ equals signal on the $i$ axis due to unit $\dot{B}$ on the $j$ axis. Then by inversion:

$$
\dot{B}=K^{-1} S,
$$

where $K^{-1}$ is the inverse of the signal/ $\dot{B}$ matrix obtained above. These matrices have been calculated for all coilsets, and are automatically applied to correct the signal by the processing software. This method completely compensates for coil misalignment, twisting of the form, and so on. The alignment is re-verified after insertion by inserting a Helmholtz coil through the gun opening. The accuracy of the signal is then only limited by the accuracy with which we can acquire signals which is better than $0.1 \%$, or the accuracy of alignment of the calibration field which is about $\theta \approx 0.5^{\circ}$. The corresponding cross talk error is:

$$
\frac{B_{\perp} \cdot d A}{B_{\|} \cdot d A}=\frac{B \sin (\theta)}{B \cos (\theta)}=0.008
$$

resulting in a total cross talk error of about $1 \%$, which should not interfere with measurements except at the wall or precisely at $z=L / 2$ where $B_{r}$ should be exactly zero. In those places, small deviations from zero are likely to be cross talk error.

Due to the short $(100 \mu \mathrm{s})$ lifetime of the spheromak, integration of the $\dot{B}$ signals to recover magnetic field is a significant problem. Signals can be integrated either with the use of analog RC integrators, or by sampling $\dot{B}$ at a high rate (with an RC filter to remove the highest frequency noise) and digitally integrating the signal in post processing. Each technique has its difficulties. Since noise is eliminated and the integrated signal changes more slowly, analog integrators allow use of slower digitizers. However, they cause loss of signal intensity and can "droop," causing signal distortion due to the discharge of the capacitor. On the other hand, digital integrators offer great precision, but since errors in the sum will propagate they require high sampling rates. SSX has 32 channels of $10 \mathrm{MHz}$ digitizers, and four at $50 \mathrm{MHz}$. The $50 \mathrm{MHz}$ digitizers are easily fast enough to digitally integrate, and the $10 \mathrm{MHz}$ units can do so with some signal processing, so this method is chosen. The $10 \mathrm{MHz}$ digitizers are "corrected" by forcing the integral (i.e., $B$ ) to zero at the beginning and at end of the run after the $\dot{B}$ signal returns to zero. This must be true physically since there is no field before or after the experiment, and it is verified by the faster digitizers. A correction is then applied to the rest of the signal to make it fit these conditions. This process produces good agreement with the faster digitizers. It is incorporated into the same automatic signal processing code which applies the cross-calibration matrix corrections. Despite these steps, however, integration is the least accurate step in our data acquisition, with possible errors as high as $10 \%$. In order to detect ground loops and possible HV shorts of the probes to the casing, the processing code also looks to make sure all the probes zero out at about the same time. A suspicious probe is flagged allowing the experimenter to evaluate it.

There is always concern, when inserting probes into the plasma, that they will disrupt it so much as to invalidate measurements. Tests in the small flux conserver seem to indicate that this is not a significant problem. Tests were first run with a small "nub" probe which extended only $1 / 4$ " into the conserver, which should have very little effect. The same type of runs were repeated with the long linear array. No shortening of lifetime was observed, and signals were similar to within shot variability limits, indicating that there was not significant disruption. Since the probes are smaller in relation to plasma size and energy in the lrge than in the small flux conserver, we expect that disruption should not be an issue there either.

\section{ACKNOWLEDGMENTS}

M. R. B. is a DOE Junior Faculty Investigator. Special thanks to P. Bellan for providing MHD lecture notes and S. Palmer for construction of the apparatus.

This work was performed under Department of Energy (DOE) Grant No. DE-FG02-97ER54422 and constitutes part of the undergraduate honors thesis of C.G.R.G. (recipient of the 1997 APS Apker Award for undergraduate physics thesis).

${ }^{1}$ M. Yamada, Y. Ono, A. Hayakawa, M. Katsurai, and F. W. Perkins, Phys. Rev. Lett. 65, 721 (1990).

${ }^{2}$ M. Yamada, F. W. Perkins, A. K. MacAulay, Y. Ono, and M. Katsurai, Phys. Fluids B 3, 2379 (1991).

${ }^{3}$ M. Yamada, H. Ji, S. Hsu, T. Carter, R. Kulsrud, Y. Ono, and F. Perkins, Phys. Rev. Lett. 78, 3117 (1997). 
${ }^{4}$ Y. Ono, A. Morita, M. Katsurai, and M. Yamada, Phys. Fluids B 5, 3691 (1993).

${ }^{5}$ Y. Ono, M. Yamada, T. Akao, T. Tajima, and R. Matsumoto, Phys. Rev. Lett. 76, 3328 (1996).

${ }^{6}$ T. R. Jarboe, Plasma Phys. Controlled Fusion 36, 945 (1994).

${ }^{7}$ M. R. Brown and A. Martin, Fusion Technol. 30, 300 (1996).

${ }^{8}$ T. K. Fowler, J. S. Hardwick, and T. R. Jarboe, Plasma Phys. Controlled Fusion 16, 91 (1994).

${ }^{9}$ E. B. Hooper, J. H. Hammer, C. W. Barnes, J. C. Fernández, and F. J. Wysocki, Fusion Technol. 29, 191 (1996).

${ }^{10}$ E. B. Hooper and T. K. Fowler, Fusion Technol. 30, 1390 (1996)

${ }^{11}$ F. J. Wysocki, J. C. Fernńdez, I. Henins, T. R. Jarboe, and G. J. Marklin, Phys. Rev. Lett. 65, 40 (1990).

${ }^{12}$ T. R. Jarboe, F. J. Wysocki, J. C. Fernńdez, I. Henins, and G. J. Marklin, Phys. Fluids B 2, 1342 (1990).

${ }^{13}$ R. E. Chrien, J. C. Fernńdez, I. Henins, R. M. Mayo, and F. J. Wysocki, Nucl. Fusion 31, 1390 (1991).

${ }^{14}$ C. W. Barnes, T. R. Jarboe, G. J. Marklin, S. O. Knox, and I. Henins, Phys. Fluids B 8, 1871 (1990).

${ }^{15}$ M. R. Brown and A. D. Bailey III, and P. M. Bellan, J. Appl. Phys. 69, 6302 (1991)

${ }^{16}$ W. C. Turner, E. H. A. Granneman, C. W. Hartman, D. S. Prono, J. Taska, and A. C. Smith, Jr., J. Appl. Phys. 52, 175 (1981)
${ }^{17}$ W. C. Turner, G. C. Goldenbaum, E. H. A. Granneman, J. H. Hammer, C. W. Hartman, D. S. Prono, and J. Taska, Phys. Fluids 26, 1965 (1983)

${ }^{18}$ M. J. Schaffer, Phys. Fluids 30, 160 (1987).

${ }^{19}$ J. B. Taylor, Phys. Rev. Lett. 33, 1139 (1974).

${ }^{20}$ J. B. Taylor, Rev. Mod. Phys. 58, 741 (1986).

${ }^{21}$ M. R. Brown, J. Plasma Phys. 57, 203 (1997).

${ }^{22}$ S. O. Knox, C. W. Barnes, G. Marklin, T. R. Jarboe, I. Henins, H. W. Hoida, and B. L. Wright, Phys. Rev. Lett. 56, 842 (1986).

${ }^{23}$ D. A. Kitson and P. K. Browning, Plasma Phys. Controlled Fusion 32, 1265 (1990)

${ }^{24}$ P. K. Browning, J. R. Clegg, R. C. Duck, and M. G. Rusbridge, Plasma Phys. Controlled Fusion 35, 1563 (1993).

${ }^{25}$ A. Bondeson, G. Marklin, Z. G. An, H. H. Chen, Y. C. Lee, and C. S. Liu, Phys. Fluids 24, 1682 (1981).

${ }^{26}$ J. M. Finn W. M. Manheimer, and E. Ott, Phys. Fluids 24, 1336 (1981).

${ }^{27}$ M. R. Brown and P. M. Bellan, Nucl. Fusion 32, 1125 (1992).

${ }^{28}$ C. W. Barnes, J. C. Fernńdez, I. Henins, H. W. Hoida, T. R. Jarboe, S. O. Knox, G. J. Marklin, and K. F. McKenna, Phys. Fluids 29, 3415 (1986).

${ }^{29}$ F. J. Wysocki, J. C. Fernández, T. R. Jarboe, and G. J. Marklin, Phys. Rev. Lett. 61, 2457 (1988).

${ }^{30}$ G. W. Hart, C. Chin-Fatt, A. W. DeSilva, G. C. Goldenbaum, R. Hess, and R. S. Shaw, Phys. Rev. Lett. 51, 1558 (1983). 